\title{
Potential patch antenna application with particle size variation in polycrystalline gadolinium iron garnet (GdIG)
}

\begin{abstract}
The effect of different particle size obtained by diverse milling time towards the microstructure and magnetic properties of gadolinium iron garnet (GdIG) at room temperature was reported in this research work. Gadolinium iron garnet (GdIG) has been mechanically alloyed at 3, 6, 9 and $12 \mathrm{~h}$ followed by sintering at $1200^{\circ} \mathrm{C}$. The samples were characterized using transmission electron microscopy (TEM), X-ray diffraction (XRD), field emission scanning electron microscopy (FESEM), vibrating sample magnetometer (VSM) and impedance analyser. All milled samples were in nanosized of $36.9,27.2,19.2$ and $20.8 \mathrm{~nm}$ for $3,6,9$ and $12 \mathrm{~h}$, respectively. The single phase of gadolinium iron garnet (GdIG) with average grain size of $0.66,0.67,0.72$ and $0.69 \mu \mathrm{m}$, respectively, showed low initial permeability and low magnetic loss when applied with low-frequency range. The effect of magnetic properties was proven to be dominantly contributed by sintering process. The result illustrated that the polycrystalline GdIG has the ability to be applied as potential patch antenna application.
\end{abstract}

Keyword: Gadolinium iron garnet (GdIG); Sintering; Permeability; Loss factor; Magnetic properties 